\title{
Prävention von Psychosen: Erwartungen erfüllt
}

\author{
Psychosis Prevention: Expectations Met
}

\section{Bibliografie}

DOI http://dx.doi.org/

10.1055/s-0030-1266056

Psychiat Prax 2011; 38: 58-60

(c) Georg Thieme Verlag KG

Stuttgart · New York.

ISSN 0303-4259

Korrespondenzadressen Priv.-Doz. Dr. med. A. Bechdolf, M. Sc.

Department of Youth Mental

Health, University of Melbourne, Australien

Klinik für Psychiatrie und Psychotherapie, Universität zu Köln

Kerpenerstraße 62

50924 Köln

andreas.bechdolf@uk-koeln.de

Prof. emer. Dr.

Reinhard Peukert

Forststraße 6

65193 Wiesbaden

reinhard.peukert@hs-rm.de

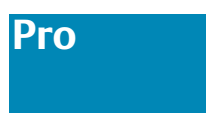

Früherkennung und Frühintervention haben die Prognose einer Reihe von Volkserkrankungen wie z.B. arterieller Hypertonie oder Diabetes mellitus wesentlich verbessert. Die schizophrene Störung zeigt in vielen Fällen einen chronischen Verlauf, kann für die Betroffenen und ihre Angehörigen mit schwerem und langjährigem Leid einhergehen und auf gesellschaftlicher Ebene erhebliche Kosten verursachen [1,2]. Vor diesem Hintergrund erscheinen Bemühungen um eine Prävention der Erkrankung dringlich notwendig.

Bei der Prävention von Psychosen, wie sie derzeit betrieben wird, handelt es sich nicht um den Versuch einer Primärprävention universal in der Allgemeinbevölkerung oder selektiv bei noch gesunden Risikoträgern, sondern um eine indizierte Prävention bei Personen, die bereits klinisch symptomatisch sind und wegen der damit verbundenen Beschwerden und Einschränkungen aktiv Hilfe suchen.

\section{Aktueller Stand zu Risikokriterien für erste psychotische Episoden}

In den letzten 15 Jahren wurden Kriterien für ein klinisch erhöhtes Psychoserisiko entwickelt, welche im Wesentlichen auf unterschwelligen psychotischen Symptomen [3] oder selbstwahrnehmbaren Denk- oder Wahrnehmungsstörungen (sog. Basissymptomen, [4]) beruhen. Diese Kriterien sind reliabel zu erheben [5, 6], wurden mittlerweile von verschiedenen Arbeitsgruppen weltweit prospektiv auch an größeren Patientenkohorten evaluiert und gehen mit einem Übergang in die Psychose von 20-30\% binnen 12 Monaten einher (z.B. $[7,8])$.

\section{Charakteristika von Personen mit erhöhtem Psychoserisiko} Personen mit erhöhtem Psychoserisiko gehen oft durch eine lange Phase frustraner Suche nach Unterstützung [9] und leiden unter klinischen Symp- tomen, die zu einer gestörten Rollenerfüllung und zu reduzierter subjektiver Lebensqualität führen [10]. Zwischen 60 und $80 \%$ der Betroffenen erfüllen zusätzlich zum Risikosyndrom Diagnosekriterien einer psychiatrischen Störung nach DSM IV, am häufigsten Depression und Angststörungen [11]. Darüber hinaus wurden eine Reihe von schizophrenieähnlichen neurobiologischen Veränderungen bei Personen mit erhöhtem Psychoserisiko beschrieben (Übersicht bei [12]).

\section{Aktueller Stand zur Frühintervention bei} Personen mit erhöhtem Psychoserisiko Im letzten Jahrzehnt haben Forschergruppen in Australien, USA und Europa Modelle für Frühinterventionen bei Personen mit erhöhtem Psychoserisiko entwickelt. Die meisten Gruppen haben hierfür spezielle Früherkennungs- und Therapiesettings etabliert, in denen mithilfe von Öffentlichkeitsarbeit Wissensvermittlung über Prodromalsymptome und weitere Indikatoren für ein erhöhtes Psychoserisiko sowie über mögliche Unterstützung und Behandlung von Betroffenen angeboten wird. Das Behandlungsangebot ist niederschwellig und nicht stigmatisierend gestaltet. Derzeit liegen 6 abgeschlossene randomisiert-kontrollierte Studien vor, welche Low-dose-Risperidon und kognitive Verhaltenstherapie [13], kognitive Verhaltenstherapie alleine [14-16], Olanzapine alleine [17] oder Omega-3-Fettsäuren [18] bei Personen mit erhöhtem Psychoserisiko evaluiert haben. Diese Studien haben zum Ergebnis, dass die Patienten der jeweiligen Experimentalbedingung ganz überwiegend einen günstigeren Symptomverlauf zeigen, eine bessere soziale Anpassung und weniger Übergänge in die Psychose aufweisen. Hierbei waren die präventiven Effekte gemessen an der Anzahl der Patienten die präventiv behandelt werden müssen um ein Ereignis zu verhindern (Number Needed to Treat - NNT) z.T. wesentlich stärker ausgeprägt als dies z. B. für die Prävention von Schlaganfällen durch antihy- 
pertensive Medikation bei Patienten mit arterieller Hypertonie bekannt ist (NNT 4 [13] vs NNT 13 [19]). Erste Untersuchungen zeigen, dass Personen mit erhöhtem Psychoserisiko, die eine Frühintervention erhalten, im Vergleich zu psychotisch Ersterkrankten ohne Frühintervention im weiteren Verlauf weniger psychiatrische Behandlung in Anspruch nehmen, sodass die Frühintervention mittelfristig kostengünstiger als die Standardbehandlung ist [20].

\section{Haben sich die Erwartungen an die Psychoseprävention erfüllt?}

Die Bemühungen um die Prävention von Psychosen haben zu prospektiv evaluierten Risikokriterien geführt, welche eine hilfesuchende Population identifiziert, die klinisch symptomatisch und funktionell beeinträchtigt ist. Diese Population zeigt ein Risiko innerhalb der nächsten 12 Monate an einer psychotischen Störung zu erkranken, welches mehrere 100-fach über dem Erkrankungsrisiko der Normalbevölkerung liegt und deren Übergangszahlen in eine psychotische Erstmanifestation klinisch bedeutsam sind. Die bisher entwickelten Interventionen führen zu einer Verbesserung der zur Behandlung führenden Symptomatik und des Funktionsniveaus und können das Auftreten psychotischer Episoden zumindest verzögern, wenn nicht sogar mittelfristig verhindern. Auch wenn die bisherigen Studien zeigen konnten, dass die klinischen Symptome, die Funktionseinschränkung und die subjektive Belastung wegen derer die Patienten Hilfe suchen durch die Frühintervention signifikant gebessert werden und diese Effekte alleine die Frühintervention rechtfertigen könnten, sind bezüglich des präventiven Aspektes der Früherkennung und Frühintervention hohe ethische Maßstäbe anzulegen. Nicht alle Betroffenen werden tatsächlich im unbehandelten Verlauf eine Psychose entwickeln und dementsprechend muss das jeweilige Aufklärungsund Hilfsangebot durch eine verantwortungsbewusste NutzenRisiko-Abwägung zu rechtfertigen sein. Alleine die Informationsvermittlung, einer Risikopopulation anzugehören, könnte potenziell angstauslösend und stigmatisierend wirken. Die Erfahrungen an den Früherkennungszentren sind jedoch, dass die empathische und individualisierte Informationsvermittlung in einem angemessenen Setting meist zur Entlastung bei den Betroffenen führt. Bezüglich der Frühinterventionen sind akzeptable, wenig stigmatisierende und nebenwirkungsarme Interventionen wie Psychotherapie und Omega-3-Fettsäuren weniger risikobehaftet als antipsychotische Medikation (Übersicht zur ethischen Diskussion in [21]).

Eine Reihe von Fragen sind noch offen: Kann die prädiktive Vorhersage der Kriterien verbessert werden? Wie sind die Ergebnisse des Langzeit-Follow-ups? Welche Intervention hat das günstigste Nutzen-Risiko-Profil? Obwohl Empfehlungen zum Umgang mit Risikopersonen und zu deren Psychotherapie vorliegen [22,23], ist der Transfer der Erkenntnisse in die Versorgungspraxis im deutschen Sprachraum sicher (noch) nicht gelungen - auch weil die Kostenträger zum jetzigen Zeitpunkt Früherkennung und Frühintervention nicht entgelten. Ein erster Schritt in Richtung einer Generalisierung der wissenschaftlichen Erkenntnisse könnte die Einführung eines Risikosyndroms in DSM-V sein, wie es derzeit diskutiert wird [24]. Der Direktor des National Institute of Mental Health in den USA, T. R. Insel, hat kürzlich festgestellt, dass sich die pharmakologische, psychosoziale und pathophysiologische Forschung der letzten 20 Jahre nicht in einer Verbesserung der Prognose von schizophrenen Störungen niedergeschlagen hat [25]. Vor diesem Hintergrund sind die Ergebnisse der Prävention von Psychosen ein Hoffnungsschimmer am Horizont.
Niemand wird ernsthafte Argumente gegen Prävention vortragen wollen. Dieses Kontra kann weder mit empirischen Gegenbelegen noch einer grundsätzlichen Argumentation gegen Prävention (von Psychosen) antreten - bestenfalls mit einigen Randbemerkungen, deren Relevanz der geneigte Leser zu entscheiden hat.

Noch im Jahre 2004 auf der Jahrestagung der Aktion Psychisch Kranke konnte Warner [26] empirisch fundiert und methodisch korrekt berechnet die Problematik von Früherkennung und -behandlung herausstellen. Anhand der Daten von Klosterkötter et al. [4] referierte er den positiven Vorhersagewert von ca. 70\% und kam zu dem Ergebnis: Würde der Test auf die Allgemeinbevölkerung ausgeweitet (und nicht nur auf eine Risikopopulation), käme man zu einem positiven Vorhersagewert von nur noch $2 \%$ - bei gleichzeitig $40 \%$ an falschen Vorhersagen einer beginnenden Schizophrenie.

Warner weiß, dass sich die Studie von Klosterkötter und die Strategien des „Vaters“ der Früherkennung, McGorry, gerade aufgrund der Gefahr der vielen falsch Positiven auf eine Risikopopulation bezogen haben. Warner monierte: so werde die Reduktion des Risikos der falsch Positiven mit einer unbekannt großen Anzahl nicht erkannter Fälle in der Gesamtbevölkerung erkauft.

Unabhängig von Warners Kritik haben die Früherkennungszentren mit ihren systematischen Erhebungsinstrumenten und den gewählten Strategien sowohl die berechenbare prädiktive Stärke verbessert als auch das Risiko von falsch Positiven deutlich begrenzt und eine Verbesserung der Identifikation von Fällen innerhalb der Gesamtbevölkerung erreicht. Das breite öffentliche Publikum (Primärprävention) wird durch Inserate und Plakate auf das Vorliegen subkutaner prämorbider Zeichen angesprochen und nach Kontaktaufnahme mit dem Zentrum als psychosenah oder psychosefern klassifiziert. Diese Trennung begrenzt das von Warner deutlich hervorgehobene „Schadenspotenzial“ dieser Strategie, denn den psychosefernen Besuchern werden psychologische und soziale Unterstützungen zuteil - aber keine pharmakologische Behandlung.

Leider liegen mir keine Daten vor, aus denen sich berechnen ließe, wie viele Menschen z.B. nach BLIPS (ein Definitionsmerkmal für das psychosenahe Stadium: kurzzeitige, intermittierende psychotische Symptome) nicht die Diagnoseschwelle zur Schizophrenie überschreiten und wie viele Menschen trotz Frühbehandlung an einer Schizophrenie erkranken. Aus diesen Zahlen ließe sich das Chancen-Risiko-Verhältnis errechnen, das jedem Nutzer eines Früherkennungs- und -behandlungszentrums mitgeteilt werden müsste, um eine informierte Entscheidung zu treffen.

Durch Frühbehandlung im psychosenahen Stadium sollen die negativen Folgen der Erkrankung abgeschwächt werden. Allerdings steht zu befürchten, dass eine Kontrolle der psychosozialen Variablen den positiven Effekt dieses früheren Behandlungsbeginns auflösen. Für diese Möglichkeiten sprechen ältere Daten von T. J. Croudence von der Universität Nottingham.

Aus meiner Sicht als Nichtpsychiater stellt sich allerdings eine ganz andere Frage.

Ist es - gesellschaftspolitisch gesehen - eine richtige Strategie, Menschen mit seelischen Belastungen, die das Risiko einer Psychose bergen, ausschließlich und sofort psychiatrie- und kliniknahen Hilfen zuzuführen? Wäre es nicht der zu Recht in der UN-Behindertenrechtskonvention geforderten Inklusionsperspektive viel angemessener, wenn die Richtung umgedreht würde - und sich die psychiatrischen, insbesondere gemeindepsychiatrischen Hilfen in die Sozialräume verlagern, dort diffundieren und sich 
mit den dort längst vorhandenen Stützungs- und Hilfestrukturen verbinden? [27]

Konkret gesagt: wenn die Tagesstätten sich zu einem Teil virtualisieren und die jetzigen Besucher von den Mitarbeiterinnen nicht alle Angebote in eigenen Räumen realisieren, sondern an gewöhnlichen Orten des Sozialraumes (Kochen - in einer Altentagesstätte oder einem Kindergarten; Reinigen - als Angebot an Gebrechliche der Region; handwerklich werken - als Hausmeisterdienst und in einem für den gesamten Stadtteil zugänglichen Handwerks- und Kreativladen etc.) - dann wären die begleitenden Fachkräfte im Sozialraum präsent und könnten sich den Bürgern der Region zu allen Fragen seelischer Gesundheit zur Verfügung stellen. Dies ließe sich für die Kontaktstellen und die Wohnbereiche genauso durchbuchstabieren [28]. Ein solcher Kontext senkt nicht nur die Schwelle für Anfragen, die psychologischen und sozialen Hilfen für Menschen in einer psychosefernen Phase ließen sich zudem mit den Angeboten für alle Bürger des Sozialraums verbinden [29]. Natürlich bedarf es eines unmittelbaren Zugangs zu fachmedizinischer Kompetenz, um die Funktion von Prävention über Früherkennung und -behandlung zu erfüllen. Dies wird zunehmend leichter werden im Rahmen von integrierter Versorgung, die vom SGB-V-Bereich oder dem Bereich der Hilfen zur Teilhabe ausgehend diese beiden Bereiche miteinander intelligent verknüpft.

Für eine konsequente Orientierung dieser und aller anderen Präventionsanstrengungen im Bereich seelischer Gesundheit im Sozialraum sind die guten Gründe Legion. Die Erkrankung an Psychosen korreliert mit prä-, peri- und postnatalen Komplikationen. Die Einführung der kontinuierlichen Begleitung durch Hebammen hat sich überall dort, wo es eingeführt wurde, bewährt. Wir wissen aber auch: der Einfluss der Geburtskomplikationen auf die psychische Gesundheit im Erwachsenenalter ist abhängig von der sozialen Umwelt im Kindesalter, vornehmlich vom Einkommen der Eltern (Fan und Eaton).

In Deutschland haben die KiGGS und BELLA-Studie gezeigt: es sind vorrangig soziale Merkmale, die ein seelisch gesundes Aufwachsen von Kindern verhindern - und psychotische Erkrankungen sind ein Teil des Resultats. Dieses Wissen hat sehr viele und vielfältige regionale Projekte veranlasst, Programme in nahezu allen Bundesländer und zudem ein BMFSJ-Aktionsprogramm „Frühe Hilfen“ [30]. Dabei geht es um die Aktivierung von Schutzfaktoren, insbesondere der Stärkung familiärer Ressourcen und die Implementation von Aufklärungs- und Unterstützungsstrukturen in alltäglichen Settings.

(Einen Überblick über die KiGGS- und BELLA-Studie sowie die Projekte und Programme siehe unter [31].)

Alles spricht dafür: Primärprävention ist wohl nur in einer sozialraumbezogenen Herangehensweise sinnvoll - unabhängig davon, ob es sich um universelle (die das große Publikum anspricht), um selektive (beim Vorliegen von Risiken oder dem Mangel an protektiven Faktoren, wie z. B. in prekären Lebensverhältnissen) oder um indizierte Primärprävention handelt (bei Personen mit minimalen Symptomen ohne Erreichen der Diagnoseschwelle: die Zielgruppe der Früherkennungs- und -behandlungszentren). Mit dieser Erkenntnis erschließt sich beiläufig auch der therapeutische, nicht nur gesellschaftspolitische Sinn von Inklusion.

\section{Literatur}

1 Van Os J, Kapur S. Schizophrenia. Lancet 2009; 374: 635-645

2 Konnopka A, Klingberg S, Wittorf A et al. Die Kosten der Schizophrenie in Deutschland. Ein systematischer Literaturüberblick. Psychiat Prax 2009; 36: 211- 218

3 Yung AR, Phillips LJ, Yuen HP et al. Psychosis prediction: 12-month follow up of a high-risk („,prodromal“) group. Schizophr Res 2003; 60: 21-23
4 Klosterkötter J, Hellmich M, Steinmeyer EM et al. Diagnosing schizophrenia in the initial prodromal phase. Arch Gen Psychiatr 2001; 58: 158164

5 Miller TJ, McGlashan TH, Rosen JL et al. Prospective diagnosis of the initial prodrome for schizophrenia based on the structured interview for prodromal syndromes: preliminary evidence of interrater reliability and predictive validity. Am J Psychiatry 2002; 159: 863-865

6 Yung AR, Yuen HP, McGorry PD et al. Mapping the onset of psychosis: the Comprehensive Assessment of At-Risk Mental States. Aust NZ J Psychiatry 2005; 39: 964-971

7 Ruhrmann S, Schultze-Lutter F, Salokangas RKR et al. Prediction of Psychosis in Adolescents and Young Adults at High Risk Results From the Prospective European Prediction of Psychosis Study. Arch Gen Psychiatry 2010; 67: 241-251

8 Cannon TD, Cadenhead K, Cornblatt B et al. Prediction of psychosis in youth at high clinical risk: a multisite longitudinal study in North America. Arch Gen Psychiatry 2008; 65: 28-37

9 Köhn D, Pukrop R, Niedersteberg A et al. Pathways to care: help-seeking behavior in first-episode psychosis. Fortschr Neurol Psychiatr 2004; 72: 635-642

10 Bechdolf A, Pukrop R, Köhn D et al. Subjective quality of life in subjects at risk for a first episode of psychosis: A comparison with first episode schizophrenia patients and healthy controls. Schizophrenia Research 2005; 79: 137-143

11 Svirskis T, Korkeila J, Heinimaa M et al. Axis-I disorders and vulnerability to psychosis. Schizophr Res 2005; 75: 439-446

12 Correll CU, Hauser M, Auther AM et al. Research in people with psychosis risk syndrome: a review of the current evidence and future directions. J Child Psychol Psychiatry 2010; 51: 390-431

13 McGorry PD, Yung AR, Phillips LJ et al. Can first episode psychosis be delayed or prevented? A randomized controlled trial of interventions during the prepsychotic phase of schizophrenia and related psychosis. Arch Gen Psychiatry 2002; 59: 921-928

14 Morrison AP, French $P$, Walford L et al. A randomised controlled trial of early detection and cognitive therapy for the prevention of psychosis in people at ultra-high risk. Br J Psychiatry 2004; 185: 291-297

15 Addington J, Epstein I, Liu L et al. A randomized controlled trial of cognitive behavioral therapy for individuals at clinical high risk of psychosis. Schizophr Res 2010 Nov 11 Doi: 10.1016/j.schres.2010.10.015

16 Bechdolf A, Wagner M, Ruhrmann S et al. Preventing progression to firstepisode psychosis in early initial prodromal states. Br J Psychiatry 2010 in press

17 McGlashan TH, Zipursky RB, Perkins D et al. A randomized, double-blind trial of olanzapine versus placebo in patients prodromally symptomatic for psychosis. Am J Psychiatry 2006; 163: 790-799

18 Amminger GP, Schäfer MR, Papageorgiou K. Long-chain omega-3 fatty acids for indicated prevention of psychotic disorders: a randomized, placebo-controlled trial. Arch Gen Psychiatry 2010; 67: 146-154

19 Lachin J. Statistical considerations in the intention-to-treat principle. Control Clinical trials 2000; 21: 167-189

20 Valmaggia LR, McCrone P, Knapp M et al. Economic impact of early intervention in people at high risk of psychosis. Psychol Med 2009; 39: 1617-1626

21 Schizophrenia Research 2001, Bd. 51 (1)

22 BechdolfA, Veith V, Güttgemanns Jet al. Kognitive Verhaltenstherapie bei Personen mit erhöhtem Psychoserisiko: Ein Behandlungsmanual. Bern: Huber; 2010

23 Bechdolf A, Juckel G. Psychoedukation bei Personen mit erhöhtem Psychoserisiko. Stuttgart: Schattauer; 2006

24 Carpenter WT. Anticipating DSM-V: should psychosis risk become a diagnostic class? Schizophr Bull 2009; 35: 841-843

25 Insel TR. Rethinking schizophrenia. Nature 2010; 468: 187-193

26 Warner S. Prävention in der Psychiatrie - was wirkt? APK u. a. Prävention bei psychischen Erkrankungen. Neue Wege in Praxis und Gesetzgebung. Bonn: Psychiatrie-Verlag; 2005: 62-90

27 Peukert $R$. Was ist „Sozialraumorientierte Gemeindepsychiatrie“? APK u.a. Kooperation und Verantwortung in der Gemeindepsychiatrie. Bonn: Psychiatrie-Verlag; 2009: 114-128

28 Peukert R. Sinn und Möglichkeiten der Prävention bei psychischen Erkrankungen. APK u. a. Prävention bei psychischen Erkrankungen. Neue Wege in Praxis und Gesetzgebung. Bonn: Psychiatrie-Verlag; 2005: 31 61

29 BarloschkyJ. Seelische Gesundheit in den Quartieren/Stadtteilen - Prävention und Zugang zu Hilfen in Bremen-Tenever. APK u. a. Kooperation und Verantwortung in der Gemeindepsychiatrie. Bonn: PsychiatrieVerlag; 2009: 137-151

30 http//www.fruehehilfen.de/1981.html

31 Fegert JM. Frühe Hilfen und die Prävention psychischer Belastungen bei Kindern und Jugendlichen. Fachvortrag in Wiesbaden 19. August 2010. http://www.apk-ev.de/Datenbank/themen/0133_Wiesbaden_ 19_8_Fegert.pdf 